\title{
Correlation of Carotid Colour Doppler and MRI Brain in Patients Suffering from Cerebrovascular Disease
}

\author{
Dr. Simranjeet Singh ${ }^{1}$, Dr. Purvi D Desai ${ }^{2}$, Dr. Neha Tyagi ${ }^{3}$
}

\begin{abstract}
Present study was conducted with an objective to find the role of carotid colour Doppler and MRI brain in patients suffering from cerebrovascular diseases. 100 patients which were diagnosed as having acute cerebral ischemia on MRI brain were analysed with carotid Doppler in gray scale, colour Doppler and then spectral waveform and closeness of the result was obtained by correlating the findings of both the investigations.
\end{abstract}

Keywords: MRI, stroke, carotid colour Doppler, TIA

\section{Aims and Objectives}

The present study was conducted with an objective to find the correlation between carotid Doppler suggesting plaques and MRI of those patients suggesting arterial ischemia/infarction. So that by correlating the findings of both investigations, it can be made out whether carotid doppler can be used as screening tool in patients with high risks of having ischemic strokes or not.

\section{Methodology}

The protocol was approved by Ethics Committee and written informed consent was obtained from each patient. The present cross sectional study was conducted in New Civil Hospital, Surat on 100 cases of acute arterial ischemia as suggested by MRI Brain via studying T1W, T2W, Diffusion weighted, STIR Sequence. After stabilization of cases, thorough gray scale examination on Carotid Doppler was done for intima media thickness, presence, location and characterization of plaques were assessed. Spectral Doppler analysis was done and measurements of Peak systolic velocity, end diastolic velocity were obtained on a frozen image.

\section{Inclusion Criteria}

Patients with symptoms of stroke and transient ischemic attack such as transient episodes of neurological dysfunction, sudden weakness or numbness, hemi-paresis, focal neurological deficits, sudden loss of consciousness, altered sensorium, aphasia, slurring of speech, diminution or loss of vision are included in the study.

\section{Exclusion Criteria}

Those cases that had evidence of posterior circulation stroke and Evidence of hemorrhagic stroke are excluded from the study.

\section{Duration of Study}

July 2016 to November 2016. Initially the patients were managed for stroke and after that they were called next day for carotid doppler evaluation. While the doppler was performed, the procedure itself, possible complications and its accuracy had been carefully explained to the patient and/or relative and written voluntary informed consent taken.

\section{Background}

Carotid artery atherosclerosis is a major cause of disabling stroke and death and it is thought to be the predominant etiology of stroke in Western society. Moreover, stroke is the third leading cause of death and the primary cause of disability in the world. "Stroke" is a generic term that describes a clinical event characterised by sudden onset of neurological deficit.

Compared with medical therapy, surgical endarterectomy or carotid stenting have been proven to decrease stroke in symptomatic patients with severe stenosis and in a very selective group of asymptomatic patients suffering of this pathology. Nevertheless, clinical assessment of stroke risk had not progressed beyond the use of luminal stenosis in spite of evidence to suggest that this is an inadequate predictor of stroke

Imaging modalities available for the diagnosis are MRI, Contrast enhanced CT, carotid doppler for imaging of atherosclerotic plaques in carotid artery and angiography. More recently, imaging studies have suggested plaque composition as an independent risk factor for ischemic stroke.

Because of this, many efforts have been made to correlate symptoms and cerebral events with histological studies, color doppler ultrasonography (CDU) and magnetic resonance imaging (MRI). MRI imaging techniques have permitted serial monitoring of atherosclerotic disease evolution and the identification of intraplaque risk factors for accelerated progressión.

According to SRU (Society of radiologists in ultrasound) consensus for diagnosis and stratification of ICA stenosis recommendations have been given which are as follows:

Normal: ICA PSV is $<125 \mathrm{~cm} / \mathrm{sec}$ and no plaque and intimal thickening visible sonographically

\section{Volume 6 Issue 12, December 2017}




\section{International Journal of Science and Research (IJSR) \\ ISSN (Online): 2319-7064}

Index Copernicus Value (2016): 79.57 | Impact Factor (2015): 6.391

$<50 \%$ Stenosis: ICA PSV is $<125 \mathrm{~cm} / \mathrm{sec}$ and intimal thickening is visible

$50-69 \%$ stenosis: ICA PSV is $125-230 \mathrm{~cm} / \mathrm{sec}$ and plaque is visible sonographically

$>70 \%$ stenosis but less then near total occlusion: ICA PSV

$>230 \mathrm{~cm} / \mathrm{sec}$ and visible plaque and luminal narrowing

Near occlusion of ICA: velocities are not used here and luminal narrowing is the only criteria that is used

Total occlusion of ICA: No detectable patent lumen, there may be increased in velocity in contralateral ICA

\section{Results}

Out of 100 patients of stroke, $61 \%$ were males and $39 \%$ were females. It was observed that $46 \%$ patients were aged 60 years or above. Middle cerebral artery territory was most frequently involved ( $92 \%$ of stroke patients) while Anterior cerebral artery territory was involved in $8 \%$. According to site, Basal Ganglia/ Internal Capsule region was the most commonly affected $(76 \%)$ site. Parietal, frontal and temporal lobes were affected in 52\%, $44 \%$ and $16 \%$ of patients respectively. The mean Intima media thickness in stroke patients $1.73 \mathrm{~mm}$. multiple plaques were seen in $44 \%$ of stroke patients.

Table 1: Intima media thickness of carotid artery

\begin{tabular}{|c|c|c|}
\hline $\begin{array}{c}\text { Intima media } \\
\text { thickness }\end{array}$ & TIA* $(\boldsymbol{\%})$ & Stroke \\
\hline$<1 \mathrm{~mm}$ & 60 & 18 \\
\hline $1-2 \mathrm{~mm}$ & 28 & 56 \\
\hline $2-3 \mathrm{~mm}$ & 8 & 14 \\
\hline $3-4 \mathrm{~mm}$ & 4 & 10 \\
\hline$>4 \mathrm{~mm}$ & 0 & 2 \\
\hline Total & 100 & 100 \\
\hline \multicolumn{2}{|c}{} \\
\hline
\end{tabular}

Types of plaques (By Gray -Weale et al)

Type I: Uniformaly echolucent with a thin echogenic cap Type II: predominantly echolucent with small area of echogenicity

Type III: Predominently echogenic with small area of echolucency

Type IV: uniformaly echogenic

Type V: could not be classified due to heavy classification

Steffen et al reported that in symptomatic arteries, for all degrees of stenosis,there was preponderance of echolucent type 1 and 2 leisons, whereas in patients without symptoms there was a preponderance of echogenic plaques type 3 and 4 .

Table 2: Grading of atherosclerotic plaques

\begin{tabular}{|c|c|c|}
\hline Types of plaques & TIA $*$ & Stroke (\%) \\
\hline Type 1 & $4(9)$ & $16(25)$ \\
\hline Type II & $6(13.6)$ & $20(31.2)$ \\
\hline Type III & $6(13.6)$ & $12(18.7)$ \\
\hline Type IV & $4(9)$ & $8(12.5)$ \\
\hline Type V & $24(54.8)$ & $8(12.5)$ \\
\hline Total & $44(100)$ & $64(100)$ \\
\hline \multicolumn{2}{|c}{} \\
\hline
\end{tabular}

Table 3: Number and Multiplicity of Plaques

\begin{tabular}{|c|c|c|}
\hline Plaques in patient & TIA $(\%)$ & Stroke \\
\hline None & $\mathbf{1 4}(\mathbf{2 8})$ & $\mathbf{8}(\mathbf{1 6})$ \\
\hline 1 & $\mathbf{2 8}(56)$ & $\mathbf{2 0}(40)$ \\
\hline 2 & $\mathbf{8}(16)$ & $\mathbf{2 2}(44)$ \\
\hline Total & $\mathbf{5 0}(100)$ & $\mathbf{5 0}(100)$ \\
\hline
\end{tabular}

Table 4: Type of plaques v/s degree of stenosis in ischemic attack patients

\begin{tabular}{|c|c|c|c|c|c|c|}
\hline \% of stenosis & \multicolumn{7}{|c|}{ Type of plaques } & Total \\
\hline & I & II & III & IV & V & \\
\hline$<50$ & $2(6.2)$ & $0(0)$ & $6(18.7)$ & $4(12.5)$ & $20(62.5)$ & 32 \\
\hline $50-69$ & $2(16.6)$ & $6(50)$ & $0(0)$ & $0(0)$ & $4(33.4)$ & 12 \\
\hline 70 OR MORE & - & - & - & - & - & - \\
\hline $\begin{array}{c}\text { NEAR } \\
\text { OCCLUSION }\end{array}$ & - & - & - & - & - & - \\
\hline $\begin{array}{c}\text { TOTAL } \\
\text { OCCLUSION }\end{array}$ & - & - & - & - & - & - \\
\hline
\end{tabular}

Table 5: Type of plaques v/s degree of stenosis in stroke patients

\begin{tabular}{|c|c|c|c|c|c|c|}
\hline$\%$ of stenosis & \multicolumn{5}{|c|}{ Type of plaques } & Total \\
\hline & I & II & III & IV & $\mathrm{V}$ & \\
\hline$<50$ & $6(33.3)$ & $2(11.1)$ & $4(22.2)$ & $4(22.2)$ & $2(11.1)$ & 18 \\
\hline $50-69$ & $6(21.4)$ & $12(42.8)$ & $4(28.2)$ & $2(7.2)$ & $4(14.2)$ & 28 \\
\hline $\begin{array}{l}70 \text { OR } \\
\text { MORE }\end{array}$ & - & $6(42.8)$ & $4(28.5)$ & $4(14.2)$ & $2(14.2)$ & 14 \\
\hline $\begin{array}{c}\text { NEAR } \\
\text { OCCLUSION } \\
\end{array}$ & - & $2(100)$ & - & - & - & 2 \\
\hline $\begin{array}{c}\text { TOTAL } \\
\text { OCCLUSION }\end{array}$ & - & - & - & - & - & 2 \\
\hline
\end{tabular}

Normal Left common carotid artery color Doppler waveform in a 45-year-old male with a history of transient ischemic attack and normal brain MRI scan study
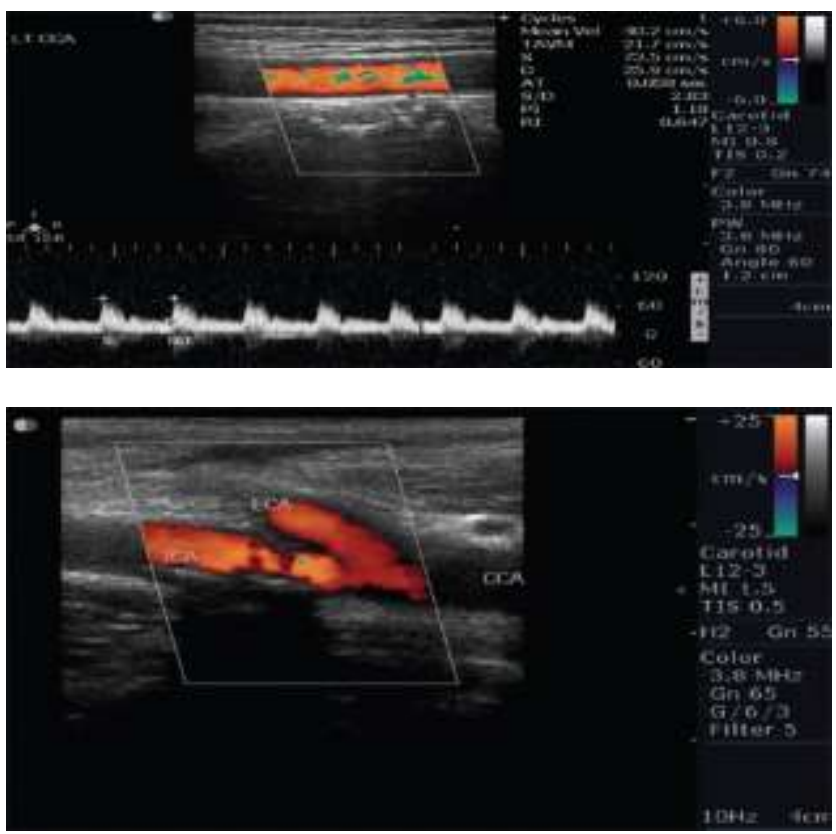

Calcific plaque at common carotid artery bifurcation in a 79-year-old female with a history of diabetes mellitus having lacunar infarcts in bilateral corona radiata 


\section{International Journal of Science and Research (IJSR) \\ ISSN (Online): 2319-7064}

Index Copernicus Value (2016): 79.57 | Impact Factor (2015): 6.391

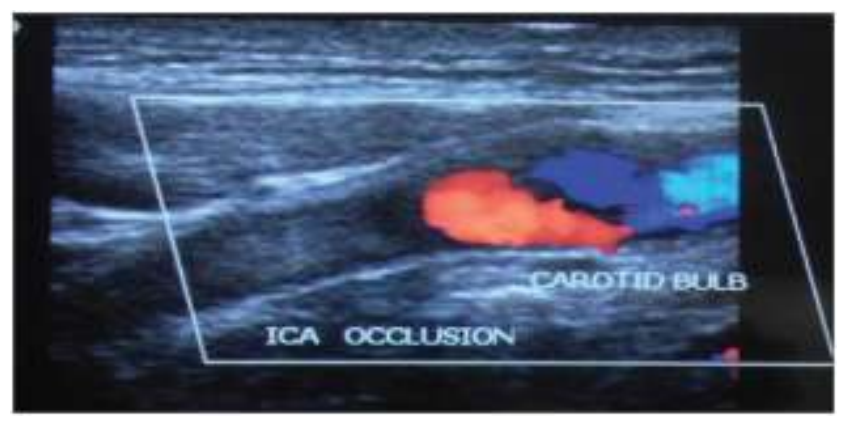

Complete occlusion of left internal carotid artery with hypoechoic plaque in a 72-year-old male with left middle cerebral artery territory infarct

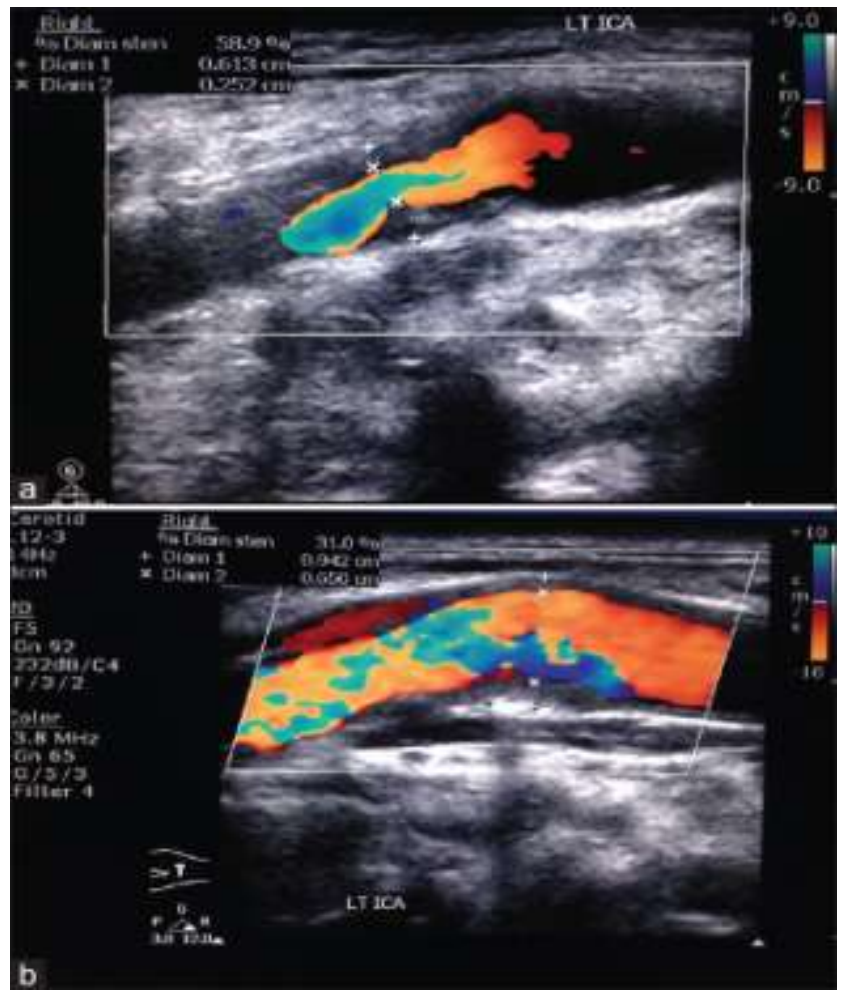

a) Soft plaque in right carotid bulb with $58.9 \%$ stenosis in a 64-year-old male having right middle cerebral artery territory infarct.

(b) Soft plaque in same patient with $31 \%$ stenosis in left proximal internal carotid artery

Internal Carotid Artery was the most common site of plaque in stroke patients $(43.7 \%)$, while it accounted for $23 \%$ of plaques in transient ischemic attack patients. The plaque surface appearance has been defined as irregular when height variation between 0.4 and $2 \mathrm{~mm}$ appeared to be present along the contour of the lesion .Most of the plaques $(82 \%)$ in Transient ischemic attack patients were smooth while in stroke patients approximately $46 \%$ plaques were smooth surfaced. Irregular plaques were much more commonly seen in stroke patients $(43.7 \%$ in stroke versus $18 \%$ in Transient ischemic attack). Ulcerated plaques were seen exclusively in stroke patients $(9.3 \%)$. Majority of plaques in stroke patients $(43.7 \%)$ caused 50 $69 \%$ stenosis.
There were minor differences in percentage of $<50 \%, 50$ $69 \%$ and 70 or more stenosis detected by colour doppler as compared to gray scale. In stroke patients, Type I was the most common type of plaque causing $<50 \%$ stenosis while TYPE II was the most common type causing 50 or more stenosis. $16 \%$ of stroke patients had no stenosis on carotid doppler evaluation. $60 \%$ of patients had ipsilateral carotid stenosis, $24 \%$ had bilateral stenosis. None had contralateral stenosis alone. Sensitivity of presence of carotid stenosis in stroke patients was $84 \%$.

Table 6: Correlation of percentage of stenosis with size of infarct

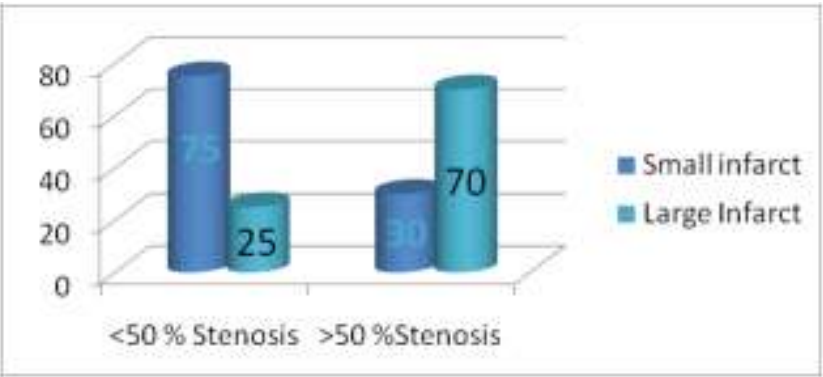

\section{Discussion}

Intima media thickness is considered as a surrogate marker for atherosclerotic disease not only in the cerebrovascular system, but in the whole arterial system. It is believed that thickening of the intima media thickness complex greater than $0.8 \mathrm{~mm}$ is abnormal and may represent the earliest changes of atherosclerotic disease. The mean intima media thickness in TIA and stroke patients was $1.1 \mathrm{~mm}$ and 1.7 mm respectively. In this study $73 \%$ and $84 \%$ of patients showed evidence of plaques in transient ischemic attack and stroke group of patients respectively. However, this also reflects the increased prevalence of carotid stenosis in ischemic stroke patients.

The multiplicity of plaques was approximately thrice in stroke patients as compared to transient ischemic attack patients. Most of the plaques $(81 \%)$ in transient ischemic attack patients were smooth surfaced while in stroke patients, irregular surfaced and ulcerated plaques together accounted for $53 \%$ of plaques. This is because embolic occlusion of intracranial arteries is the primary cause of stroke and denuded or ulcerated carotid plaque surfaces are common sources of cerebral embolism IPH was not only identified in most symptomatic patients but also a close relationship was established between the onset of symptoms and the presence of plaque haemorrhage.

Majority of the plaques $(72.7 \%)$ caused $<50 \%$ stenosis in transient ischemic attack patients while the majority $(43.7 \%)$ caused $50-69 \%$ stenosis in stroke patients. This is because intra-plaque haemorrhage and ulceration are considered unstable and lead to abrupt increases in plaque size. So larger plaques (stenosis greater than 70\%) were mostly Types 1 and 2 and typically found in symptomatic patients. One patient misdiagnosed as total occlusion by colour Doppler was correctly diagnosed as near occlusion (with streak of residual lumen: string sign) on power Doppler. 


\section{International Journal of Science and Research (IJSR) \\ ISSN (Online): 2319-7064}

Index Copernicus Value (2016): 79.57 | Impact Factor (2015): 6.391

\section{Conclusion}

The present study has shown that Carotid Doppler is an important non invasive diagnostic tool. Sensitivity of presence of carotid stenosis in stroke patients was $84 \%$ hence it can be used for screening in high risk asymptomatic patients, patients with history of cerebrovascular events and for determining treatment protocol. Thus it should be used as a first line screening investigation in these patients

\section{References}

[1] Whitlow PL, Lylyk P; Londero H Mendiz, OA; Mathias K, Jaeger H, Parodi, J, Schönholz C, Milei, J. Carotid Ar- tery Stenting Protected With an Emboli Containment System. Stroke. 2002;33:1308-1314.

[2] The global burden of disease: 2004 update. World Health Organization (WHO)Press; 2008. http://www.nhlbi.nih.gov/health/healthtopics/topics/catd/ [Online]

[3] North American Symptomatic Carotid Endarterectomy Trial Collaborators. Beneficial effect of carotid endarte- rectomy in symptomatic patients with high-grade carotid stenosis. N Engl J Med. 1991;325: 445-453.

[4] Paraskevas KI, Mikhailidis DP, Moore WS, Veith FJ. Optimal contemporary management of symptomatic and asymptomatic carotid artery stenosis. Vascular. 2011;19(3): 117-20.

[5] Inzitari D, Eliasziw M, Gates P, et al, North American Symptomatic Carotid Endarterectomy Trial Collabora- tors. The causes and risk of stroke in patients with asymptomatic internal-carotidartery stenosis. New Eng- land Journal of Medicine 2000;342:1693e700.

[6] Golledge J, Greenhalgh RM, Davies AH. The sympto- matic carotid plaque. Stroke 2000;31:774e81. 7. Watanabe Y, Nagayama M. MR plaque imaging of the carotid artery. Neuroradiology. 2010;52(4):253-74 